The intense spot in the middle of the ring is the anode spot, while the cathode glow is only partly shown at the other end. The difference in colour at different portions of the discharge tube was most characteristic, both the anode spot and the straight glow proceeding from it being yellow, the cathode glow yellow mixed with pink, while the ring at the anode was bright pink.

The interesting feature of the experiment is that a spectrographic study has shown that whereas the anode spot gives mainly the atomic (line) spectrum of helium, the pink ring is very rich in the molecular (band) spectrum of helium.

It has been known that the band spectrum of helium comes out strongly when a condensed discharge is passed through a helium tube having a fairly wide bore and filled at the proper pressure,

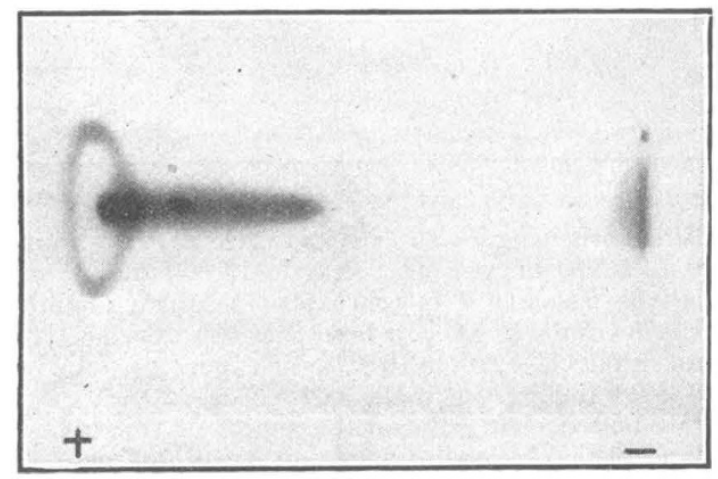

F'T. 1.

or in the cathode glow of a D.c. discharge where the yellow colour of helium is mixed with pink, but the present case of the appearance of a pink ring, only when the magnetic field is put on, seems to be novel and may provide us with another way of getting a fairly strong source of light for the study of the band spectrum of helium.

Details of the experiment will be published soon in the Scientific Papers of this Institute.

$$
\begin{aligned}
& \text { T. Takamine. } \\
& \text { T. SUga. }
\end{aligned}
$$$$
\text { A. Yanaginara. }
$$

The Institute of Physical and

Chemical Research,

Komagome, Hongo, Tokyo. July 12.

${ }^{1}$ T. Takamine, T. Suga and A. Yanagihara, NATURE, 131, 584, April 22,1933 .

${ }^{2}$ T. Takamine, T. Suga and A. Yanagihara, Sci. Pap. Inst. Phys. Chem. Res., 425, 26; 1933.

\section{Lattice Distortion and Fibre Structure in Metals}

IN recently published work $^{1}$, I have shown that the cold-working of most metals produces, first, a stage of crystal disruption which is accompanied by growth of lattice distortion, and then, after the lattice distortion limit is reached, the second stage in which the crystallites take up a preferred orienta. tion. Normally, therefore, a metal which possesses this fibre structure also exhibits lattice distortion.

It is now found that as the temperature of the metal is raised, the removal of lattice distortion takes place prior to the removal of fibre structure; and that this intermediate state, in which fibre structure exists to the exclusion of lattice distortion, persists as the metal is slowly cooled.

The observation may explain the unsuccessful attempts of many workers to establish a relation between the changes in properties induced in a metal by cold-working and the onset of fibre structure. For the properties of a metal which possesses a fibre structure will differ according to the presence or absence of lattice distortion. Thus, in the present cases, disappearance of distortion was accompanied by marked diminution of the work-hardening, although the fibre structure remained. Also, the work modifies a common assumption ${ }^{2}$ by showing that the state of fibre structure does not necessarily represent a state intermediate between that of the normal metal with its randomly oriented grains, and that of the large metallic crystal. The properties of the fibre structure state are, normally, bound up with the presence of a marked lattice distortion from which the large perfect crystal is free.

Lattice distortion is used here to denote the factor which produces diffusion of the X-ray spectral lines, as in the case of cold-worked copper, and also that which causes an abnormal weakening of the relative intensity of high order spectra, examples of which are provided by aluminium and nitrided steels.

W. A. Wood.

The National Physical Laboratory, Teddington, Middlesex. July 31 .

1 Phil. Mag., 14, 656; 1932.

2 For example, Drier and Eddy, Trans. Am. Inst. Met. Eng., p. $140 ; 1930$.

Photoelectric Absorption of $\boldsymbol{\gamma}$-Rays by Heavy Elements

THE photoelectric effect for $\gamma$-rays in heavy elements has not yet been treated satisfactorily, as it has always been found necessary to assume that $Z / 137 \ll 1$, where $Z$ is the atomic number, or else that the wave function of the photoelectron could be replaced by that of a free electron. These approximations render the result rather uncertain, and, in view of the problem of nuclear absorption, it is very desirable to have some calculation of the photoelectric effect without any uncontrolled approximation.

It is possible to do this by analysing the plane wave, representing the $\gamma$-rays, into its component spherical waves. These waves induce transitions to final states where the electron is free and the state characterised by an azimuthal quantum number $k$. The integrals representing the number of electrons in each state may then be calculated numerically by an extension of the method used in calculating the internal conversion coefficient of RaC. ${ }^{1}$

The numerical work is considerable, and so far only one case has been treated-that for $h \nu=11 \cdot 3 \times$ $10^{5}$ e.v., $Z=84$. We find for the coefficient of absorption

$$
\tau=4.0 \times 10^{-24} \mathrm{~cm} .^{2} \text { for the } K \text {-shell. }
$$

To compare this with experiment we shall use the result that $\tau$ per $K$ electron $\sim Z^{4}$ approximately.

This gives for lead

$$
\tau K \text {-shell }=3.6 \times 10^{-24} \mathrm{~cm}^{2} \quad \text { (theoretical) }
$$

The experimental value, per atom, taken from L. H. Gray's empirical formula ${ }^{2}$ is

$$
\tau \text { per atom }=4.9 \times 10^{-24} \mathrm{~cm}^{2}
$$

The chief difficulty in comparing the two is to know what fraction of the absorption is due to the $K$-shell. Rutherford, Chadwick and Ellis ${ }^{3}$ state that the $L$ electrons absorb between $\frac{1}{4}$ and $\frac{1}{b}$ as much as the $K$ electrons, and we shall assume here that about $\frac{4}{5}$ of the total absorption is due to $K$ electrons, as the relative importance of the outer electrons seems to 